\title{
WHEN IS A NON-SELF-ADJOINT HILL OPERATOR A SPECTRAL OPERATOR OF SCALAR TYPE?
}

\author{
FRITZ GESZTESY AND VADIM TKACHENKO
}

\begin{abstract}
We derive necessary and sufficient conditions for a one-dimensional periodic Schrödinger (i.e., Hill) operator $H=-d^{2} / d x^{2}+V$ in $L^{2}(\mathbb{R})$ to be a spectral operator of scalar type. The conditions demonstrate the remarkable fact that the property of a Hill operator being a spectral operator is independent of smoothness (or even analyticity) properties of the potential $V$.
\end{abstract}

RÉSUMÉ. Quand un opérateur de Hille non-autoadjoint est-il un operateur spéctral de type scalaire? Nous derivon des conditions nécessaires et suffisantes pour qur l'opérateur de Schrödinger (i.e., l'opérateur de Hill) $H=$ $-d^{2} / d x^{2}+V$ dans $L^{2}(\mathbb{R})$ soit un opérateur spéctral de type scalaire. Les conditions montrent que cette propriétés ne dépend pas des propriétés différentieles (ou analytiques) du potentiel $V$.

We consider (maximally defined) Hill operators $H=-\frac{d^{2}}{d x^{2}}+V, x \in \mathbb{R}$, in $L^{2}(\mathbb{R})$ with complex-valued $\pi$-periodic potentials $V$ such that $V \in L^{2}([0, \pi])$.

Then two fundamental problems, central to the spectral theory of such operators, are the following:

(I) What is the spectrum, $\sigma(H)$, of $H$, in particular, what is the nature and geometry of $\sigma(H)$ ?

(II) What kind of spectral decomposition (or resolution of the identity), if any, is generated by $H$ in the space $L^{2}(\mathbb{R})$ ?

Both problems are completely solved for the case of real-valued potentials, that is, for self-adjoint Hill operators. As to the first problem, the Floquet-Bloch theory states that for every such operator there exists a sequence of real numbers

$$
\lambda_{0}^{+}<\lambda_{1}^{-} \leq \lambda_{1}^{+}<\cdots<\lambda_{k}^{-} \leq \lambda_{k}^{+}<\cdots
$$

such that the spectrum of $H$ is purely absolutely continuous and has the form

$$
\sigma(H)=\bigcup_{k=0}^{\infty}\left[\lambda_{k}^{+}, \lambda_{k+1}^{-}\right]
$$

A solution of the second problem for self-adjoint Hill operators was provided by Titchmarsh [10] around 1950. He obtained an explicit formula for the resolution of the identity generated by $H$ in terms of the fundamental matrix

$$
U(z, x)=\left(\begin{array}{cc}
\theta(z, x) & \phi(z, x) \\
\theta^{\prime}(z, x) & \phi^{\prime}(z, x)
\end{array}\right), \quad U(z, 0)=I_{2}, z \in \mathbb{C},
$$

Based upon work supported by the US National Science Foundation under Grant No. DMS0405526 and the Research Council and the Office of Research of the University of MissouriColumbia.

Scientific Field: Ordinary Differential Equations. 
of solutions of the differential equation

$$
-y^{\prime \prime}(z, x)+V(x) y(z, x)=z y(z, x),
$$

where prime / denotes the derivative with respect to $x \in \mathbb{R}$.

According to Titchmarsh [10], for every element $f \in L^{2}(\mathbb{R})$, the orthogonal $L^{2}$ representation

$$
f(x)=\sum_{k=0}^{\infty} \frac{(-1)^{k}}{2 \pi} \int_{\lambda_{k}^{+}}^{\lambda_{k+1}^{-}} \frac{d \lambda}{\sqrt{1-\Delta_{+}(\lambda)^{2}}} \Phi(\lambda, x ; f)
$$

holds with

$$
\begin{aligned}
\Phi(\lambda, x ; f)= & \phi(\lambda, \pi) \theta(\lambda, x) F_{1}(\lambda)-\theta^{\prime}(\lambda, \pi) \phi(\lambda, x) F_{2}(\lambda) \\
& -\Delta_{-}(\lambda) \theta(\lambda, x) F_{2}(\lambda)-\Delta_{-}(\lambda) \phi(\lambda, x) F_{1}(\lambda), \quad \lambda \in \sigma(H),
\end{aligned}
$$

where $\Delta_{ \pm}(z)=\left[\theta(z, \pi) \pm \phi^{\prime}(z, \pi)\right] / 2, z \in \mathbb{C}, \sqrt{1-\Delta_{+}(\lambda)^{2}} \geq 0$, and $F_{1}(\lambda)=$ $\int_{\mathbb{R}} d y f(y) \theta(\lambda, y), F_{2}(\lambda)=\int_{\mathbb{R}} d y f(y) \phi(\lambda, y)$, for $\lambda \in \sigma(H)$. As a corollary, for every closed Borel set $\sigma \subset \sigma(H)$ the operator $P(\sigma)$ defined by

$$
(P(\sigma) f)(x)=\frac{1}{2 \pi} \int_{\sigma} \frac{d \lambda}{\sqrt{1-\Delta_{+}(\lambda)^{2}}} \Phi(\lambda, x ; f)
$$

is an orthogonal projection of $L^{2}(\mathbb{R})$ onto the closed subspace $\operatorname{ran}(P(\sigma))$ invariant with respect to $H$, such that the spectrum of its restriction to $\operatorname{ran}(P(\sigma))$ is contained in $\bar{\sigma}$.

While the case of self-adjoint Hill operators is under complete control, the case of non-self-adjoint Hill operators offers a remarkable complexity. The first result in this context obtained by Serov [9] reads:

The spectrum of a Hill operator with a complex-valued potential $q \in L^{2}([0, \pi])$ coincides with the set $\sigma(H)=\left\{\lambda \in \mathbb{C} \mid \Delta_{+}(\lambda) \in[-1,1]\right\}$. According to this result the spectrum $\sigma(H)$ of $H$ is formed by a system of analytic arcs in the complex plane that may intersect in inner points as shown in [7] (see also [4]). For necessary and sufficient conditions on a set $\Sigma \subset \mathbb{C}$ to be the spectrum of some periodic Schrödinger operator $H$ with periodic potential $q \in L_{\text {loc }}^{2}(\mathbb{R})$ in terms of a certain class of Riemann surfaces, we refer to [12].

While the spectrum of non-self-adjoint Hill operators has been understood since 1960, much less was known about the spectral decompositions generated by such non-self-adjoint operators. At first this appears to be unusual since all ingredients of the Titchmarsh formula (3) are also present in the non-self-adjoint situation and hence it would seem natural to use them for a corresponding spectral analysis of the non-self-adjoint case. Such attempts, however, immediately meet essential obstacles.

According to general principles of spectral analysis, to adjust the formula (3) to the non-self-adjoint case one replaces the intervals $\left[\lambda_{k}^{+}, \lambda_{k+1}^{-}\right]$by the spectral arcs comprising $\sigma(H)$ and instantly meets the first difficulty at the points of intersection of such arcs. If $\lambda_{0}$ is such a point and $\bullet$ denotes the derivative with respect to $z$, then a change of variables from $\lambda \in\left[\lambda_{k}^{+}, \lambda_{k+1}^{-}\right]$(now denoting a spectral arc) to $t \in[0,2 \pi]$, using $\Delta_{+}(\lambda)=\cos (t)$, leads to integrals of the type $\int_{T} d t\left(\Delta_{+}^{\bullet}(\lambda(t))\right)^{-1} \Phi(\lambda(t), x ; f)$, where $T \subseteq[0,2 \pi]$ is an interval containing $t_{0}$. Convergence of these integrals for arbitrary $f \in L^{2}([0, \pi])$ and their operator properties depend on the behavior of 
the meromorphic functions

$$
\frac{\phi(z, \pi)}{\Delta_{+}^{\bullet}(z)}, \quad \frac{\theta^{\prime}(z, \pi)}{\Delta_{+}^{\bullet}(z)}, \quad \frac{\Delta_{-}(z, \pi)}{\Delta_{+}^{\bullet}(z)}
$$

participating in (4).

Clearly, Hill operators $H$ commute with the operator of translation by $\pi$. McGarvey [5] initiated the study of general operators of such a type within the framework of the theory of spectral operators in the sense of Dunford [1]. A closed operator $T$ with domain $\operatorname{dom}(T) \subseteq \mathcal{H}$ is called a spectral operator if there exists a countably additive projection-valued measure $E_{T}(\cdot)$ defined on the Borel subsets $\mathcal{B}$ of $\mathbb{C}$ such that

1. $E_{T}\left(\Lambda_{1}\right) E_{T}\left(\Lambda_{2}\right)=E_{T}\left(\Lambda_{1} \cap \Lambda_{2}\right), E_{T}(\emptyset)=0, E_{T}(\sigma(T))=I_{\mathcal{H}}$.

2. $\left\|E_{T}(\Lambda)\right\| \leq C$ with $C$ independent of $\Lambda \in \mathcal{B}$.

3. $E_{T}(\Lambda) \operatorname{dom}(T) \subseteq \operatorname{dom}(T), T E_{T}(\Lambda) f=E_{T}(\Lambda) T f, f \in \operatorname{dom}(T), \Lambda \in \mathcal{B}$.

4. $E_{T}(\Lambda) \mathcal{H} \subseteq \operatorname{dom}(T)$ for $\Lambda$ bounded, $\sigma\left(\left.T\right|_{E_{T}(\Lambda) \mathcal{H} \cap \operatorname{dom}(T)}\right) \subseteq \bar{\Lambda}$.

A spectral operator $T$ is a spectral operator of scalar type if

$$
\begin{aligned}
& \operatorname{dom}(T)=\left\{g \in \mathcal{H} \mid \lim _{\cdot n \uparrow \infty} \int_{\{\lambda \in \mathbb{C}|| \lambda \mid \leq n\}} \lambda d\left(E_{T}(\lambda) g\right) \text { exists in } \mathcal{H}\right\} \\
& T f=\text { l.i.m. } n \uparrow \infty \\
& \int_{\{\lambda \in \mathbb{C}|| \lambda \mid \leq n\}} \lambda d\left(E_{T}(\lambda) f\right), \quad f \in \operatorname{dom}(T) .
\end{aligned}
$$

McGarvey was able to apply his results only to operators of the form $-d^{2} / d x^{2}+$ $p(x) d / d x+q(x), x \in \mathbb{R}$, with $\pi$-periodic functions $p$ and $q$ under the restriction $\operatorname{Im}\left(\int_{0}^{\pi} d x p(x)\right) \neq 0$. The spectra of such operators outside a sufficiently large disc are composed of some separated ovals, permitting McGarvey to prove that these operators are in some sense asymptotically spectral operators ([5], Part II). Such results ignore the existence of local spectral singularities and, at any rate, are not applicable to Hill operators $H$.

Meiman [6] noted that zeros of $\Delta_{+}^{\bullet}(\lambda)$ are integrable singularities for the functions (6) with $\Delta_{+}(z)=\cos (t)$, but this is generally incorrect for $t \in\{0, \pi, 2 \pi\}$. Another claim of the same paper is that "the crude asymptotic estimates for $\phi(\lambda, \pi)$, $\Delta_{+}^{\bullet}(\lambda)$ and the Floquet solutions of $H$ imply that the Fourier integral theory remains essentially valid also for expansions in eigenfunctions of a non-self-adjoint Schrödinger (=Hill) operator with a complex-valued potential." However, such a vague statement, without explanations as to what type of convergence and what type of function space is meant, is unsatisfactory, especially, taking into account Theorems 1-3 stated below.

Finally, we mention that Veliev [13] erroneously concluded that the spectra of Hill operators always have non-intersecting analytic arcs which invalidates some his results concerning spectral singularities and spectral expansions.

Given this incomplete and, in part, quite confusing state of affairs on the question of whether or not a Hill operator is a spectral operator of scalar type, after more than 40 years since the problem first arose, we present its solution in terms of certain functions related to equation (2). For detailed proofs of Theorems 1-3 we refer to [3]. 
Theorem 1. A Hill operator $H$ is a spectral operator of scalar type if and only if the estimates

$$
\left|\frac{\phi(\lambda, \pi)}{\Delta_{+}^{\bullet}(\lambda)}\right| \leq C, \quad\left|\frac{\theta^{\prime}(\lambda, \pi)}{(|\lambda|+1) \Delta_{+}^{\bullet}(\lambda)}\right| \leq C, \quad\left|\frac{\Delta_{-}(\lambda)}{(\sqrt{|\lambda|}+1) \Delta_{+}^{\bullet}(\lambda)}\right| \leq C
$$

hold for all $\lambda \in \sigma(H)$, with $C$ a finite positive constant independent of $\lambda \in \sigma(H)$.

If the conditions (7) are satisfied, then the functions (6) are analytic for $z$ in an open neighborhood of $\sigma(H)$.

Some disadvantage of the above conditions lies in the fact that the numerators of the fractions in (7) are not independent functions. To remedy this, we mention that Sansuc and Tkachenko [8] gave a parametrization of Hill operators using the functional parameters $\left\{\phi(z, \pi), \Delta_{+}(z), \Delta_{-}(z)\right\}_{z \in \mathbb{C}}$. The following criterion is stated in terms of these parameters.

Theorem 2. A Hill operator $H$ is a spectral operator of scalar type if and only if the following conditions $(i)$ and $($ ii) are satisfied:

(i) The function

$$
\frac{\Delta_{+}(z)^{2}-1-\Delta_{-}(z)^{2}}{\phi(z, \pi) \Delta_{+}^{\bullet}(z)}
$$

is analytic for $z$ in an open neighborhood of $\sigma(H)$.

(i) The inequalities

$$
\left|\frac{\phi(\lambda, \pi)}{\Delta_{+}^{\bullet}(\lambda)}\right| \leq C, \quad\left|\frac{\Delta_{-}(\lambda)}{(\sqrt{|\lambda|}+1) \Delta_{+}^{\bullet}(\lambda)}\right| \leq C, \quad \lambda \in \sigma(H),
$$

are satisfied with $C$ a finite positive constant independent of $\lambda \in \sigma(H)$.

If both conditions (8) and (9) are satisfied, and a point $\lambda_{0} \in \sigma(H)$ satisfies $\Delta_{+}^{\bullet}\left(\lambda_{0}\right)=0$, then $\Delta_{+}\left(\lambda_{0}\right)^{2}-1=\Delta_{-}\left(\lambda_{0}\right)=\Delta_{+}^{\bullet}\left(\lambda_{0}\right)=0, \Delta_{+}^{\bullet \bullet}\left(\lambda_{0}\right) \neq 0$, implying that the spectrum of a Hill operator, which is a spectral operator of scalar type, is formed by a system of countably many, non-intersecting, analytic arcs. The latter may degenerate into finitely many simple analytic arcs and a simple analytic semi-infinite arc, all of which are non-intersecting. Both conditions in (9) are independent; neither one of them implies the other. Moreover, analyticity of the function (8) does not follow from the estimates (9).

To prove Theorems 1 and 2 we use, similar to the paper by Tkachenko [11], the method of direct integral decompositions following Gel'fand [2], connecting the Hill operator $H$ with the family of densely defined, closed, linear operators $H(t), t \in[0,2 \pi]$ in $L^{2}([0, \pi])$ defined by the differential expression $-d^{2} / d x^{2}+q(x)$ restricted to $x \in[0, \pi]$ and the $t$-dependent boundary conditions $y(\pi)=e^{i t} y(0)$, $y^{\prime}(\pi)=e^{i t} y^{\prime}(0)$. The spectrum of the operator $H(t), t \in[0,2 \pi]$, is given by

$$
\sigma(H(t))=\left\{E_{k}(t)\right\}_{k \in \mathbb{N}_{0}}=\left\{z \in \mathbb{C} \mid \Delta_{+}(z)=\cos (t)\right\},
$$

and the spectrum of $H$ is then given by

$$
\sigma(H)=\bigcup_{0 \leq t \leq \pi} \sigma(H(t)) .
$$

For obvious reasons, the spectrum $\left\{E_{k}(0)\right\}_{k \in \mathbb{N}_{0}}$ of $H(0)$ and $\left\{E_{k}(\pi)\right\}_{k \in \mathbb{N}_{0}}$ of $H(\pi)$ is called the periodic and anti-periodic spectrum of $H$, respectively.

The following criterion involves the spectrum $\sigma(H)$, the periodic and the antiperiodic spectra $\left\{\lambda_{0}^{+}, \lambda_{k}^{ \pm}\right\}_{k \in \mathbb{N}}=\left\{E_{k}(0), E_{k}(\pi)\right\}_{k \in \mathbb{N}_{0}}$ of $H$, the Dirichlet spectrum 
$\left\{\mu_{k}\right\}_{k \in \mathbb{N}}$, the set of critical points $\left\{\delta_{k}\right\}_{k \in \mathbb{N}}$ of $\Delta_{+}$, and is connected with the algebraic and geometric multiplicities of the eigenvalues in the sets $\sigma(H(t)), t \in[0, \pi]$.

Theorem 3. A Hill operator $H$ is a spectral operator of scalar type if and only if the following conditions ( $i)-($ iii) are satisfied:

(i) Every multiple point of either the periodic or anti-periodic spectrum of $H$ is a point of its Dirichlet spectrum.

(ii) For all $t \in[0,2 \pi]$ and all $E_{k}(t) \in \sigma(H(t)$ ), each root function (i.e., element of the algebraic eigenspace) of the operator $H(t)$ associated with $E_{k}(t)$ is an eigenfunction of $H(t)$. In particular, the geometric and algebraic multiplicity of each eigenvalue $E_{k}(t)$ of $H(t)$ coincide.

(iii) Let $\mathcal{Q}=\left\{k \in \mathbb{N} \mid \operatorname{dist}\left(\delta_{k}, \sigma(H)\right) \neq 0\right\}$, then

$$
\sup _{k \in \mathcal{Q}} \frac{\left|\lambda_{k}^{+}-\lambda_{k}^{-}\right|}{\operatorname{dist}\left(\delta_{k}, \sigma(H)\right)}<\infty, \quad \sup _{k \in \mathcal{Q}} \frac{\left|\delta_{k}-\lambda_{k}^{ \pm}\right|}{\operatorname{dist}\left(\delta_{k}, \sigma(H)\right)}<\infty .
$$

Our Theorems 1-3 give three equivalent criteria for the eigenfunction system of the operators $H(t)$ to be a Riesz basis uniformly with respect to $t \in[0,2 \pi]$. The latter is equivalent for the operator $H$ to be a spectral operator of scalar type.

\section{REFERENCES}

[1] N. Dunford and J. T Schwartz, Linear Operators, Part III: Spectral Operators, WileyInterscience, New York, 1988.

[2] I. M. Gel'fand, Expansion in characteristic functions of an equation with periodic coefficients, Doklady Akad Nauk SSSR 73, 1117-1120 (1950). (Russian.)

[3] F. Gesztesy and V. Tkachenko, A criterion for Hill operators to be spectral operators of scalar type, in preparation.

[4] F. Gesztesy and R. Weikard, Floquet theory revisited, in Differential Equations and Mathematical Physics, I. Knowles (ed.), International Press, Boston, 1995, pp. 67-84.

[5] D. McGarvey, Operators commuting with translations by one. Part I, J. Math. Anal. Appl. 4,366-410 (1962), Part II, ibid 11, 564-596 (1965); Part III, ibid 12, 187-234 (1965).

[6] N. N. Meiman, The theory of one-dimensional Schrödinger operators with a periodic potential, J. Math. Phys. 18, 834-848 (1977).

[7] L. A. Pastur and V. A. Tkachenko, Geometry of the spectrum of the one-dimensional Schrödinger equation with a periodic complex-valued potential, Math. Notes 50, 1045-1050 (1991).

[8] J.-J. Sansuc and V. Tkachenko, Spectral parametrization of non-selfadjoint Hill's operators, J. Diff. Eq. 125, 366-384 (1996).

[9] M. I. Serov, Certain properties of the spectrum of a non-selfadjoint differential operator of the second order, Sov. Math. Dokl. 1, 190-192 (1960).

[10] E. C. Titchmarsh, Eigenfunction problems with periodic potentials, Proc. Roy. Soc. London A 203, 501-514 (1950).

[11] V. A. Tkachenko, Spectral analysis of the one-dimensional Schrödinger operator with periodic complex-valued potential, Sov. Math. Dokl. 5, 413-415 (1964).

[12] V. A. Tkachenko, Spectra of non-selfadjoint Hill's operators and a class of Riemann surfaces, Ann. Math. 143, 181-231 (1996).

[13] O. A. Veliev, Spectrum and spectral singularities of differential operators with complex-valued periodic coefficients, Diff. Eqs. 19, 983-989 (1983).

Department of Mathematics, University of Missouri, Columbia, MO 65211, USA

E-mail address: fritz@math.missouri.edu

URL: http://www.math.missouri.edu/personnel/faculty/gesztesyf.html

Department of Mathematics, Ben Gurion University of the Negev, Beer-Sheva 84105, ISRAEL

E-mail address: tkachenk@cs.bgu.ac.il 